\title{
PROKLA-Redaktion
}

\section{Caso oder Caos Italiano?}

Die legasthenische Vertauschung von Buchstaben charakterisiert einigermaßen präzis die historische Entwicklungsbahn des "Falls Italien «, allerdings nicht nur in einer Richtung. Daher das Fragezeichen hinter dem Titel. Vom »caos italiano« ist derzeit viel die Rede, nach Berlusconis arrogantem und gescheitertem Versuch, die komplexe italienische Gesellschaft wie ein Untemehmen zu managen. Die Berlusconi ablösende „ÜbergangsRegierung« von Technokraten, Sachwaltern von Sachzwängen, könnte da eher den Fall Italien als Lehrstïck der Krisenbewältigung inszenieren. Übergang - wohin? Der »caso italiano«, ein europäischer Fall?

Hierzulande wurde Italien als ein »caso italiano « schon in den 70er Jahren wahrgenommen, in Regierungskreisen ebenso wie in linken Studienzirkeln. Helmut Schmidt wollte Italien aus dem Arcanum der NATO verbannen, sollten die Kommunisten, wie es zeitweise in den 70er Jahren aussah, in eine Regierung eintreten. Doch das Machtkartell der Christdemokratie war in jener Phase (und während der 80er Jahre) stark und abweisend genug, um fast unangefochten $\mathrm{zu}$ regieren oder zumindest mitzuregieren und die Kommunistische Partei vor den Pforten von Palazzo Chighi oder Quirinale auszusperren. Die italienische Zivilgesellschaft entzog sich dem Griff der Christdemokratie erst in den dramatischen ersten 90er Jahren, als die Richter und Staatsanwälte der »mani pulite das politische Erdbeben auslösten, das die so stabile italienische »Partitocrazia in atemberaubender Geschwindigkeit zum Einsturz brachte. Die weitreichende Verfilzung von Wirtschaft, Politik und Mafia wurde aufgedeckt und auch strafrechtlich verfolgt. Bekannte Minister, Bürgermeister und Unternehmer landeten im Untersuchungsgefängnis und schließlich auf der Anklagebank. Gegen starke Widerstände der alten Nomenklatura trug dieser Prozeß nicht unwesentlich zur Diskreditierung der alten Parteioligarchie, vor allem der Christdemokraten und der Sozialisten bei.

In der Trümmerlandschaft der politischen Parteien entwickelte sich der einstige caso italiano zu einem scandalo italiano und vielleicht zum caos italiano: Zum ersten Mal in der westeuropäischen Nachkriegsgeschichte wurden Neofaschisten in ein Regierungsbündnis aufgenommen. Das Land, das die Kultur der »Resistenza« mehr bewahrt hatte als irgendein anderes bis zum zweiten Weltkrieg faschistisch beherrschtes Land, übergibt Faschisten einen Teil der Staatsmacht, läßt sie im »Unternehmen Italien« die Geschäfte führen, obwohl sich noch vor wenigen Jahren alle Beobachter der italienischen Szene darin einig waren, daß die Neofaschisten nicht geschäftsfähig sind.

Italien, das ist das Land, wo die Zitronen 
blühen und das in den vergangenen Jahren immer wieder Überraschungen gebracht hat, die nicht nur liebgewordene Interpretationen sehr schnell recht alt aussehen ließen, sondern neue Analysen provozierten und einer zerknirschten Linken strategisches Umdenken abverlangte. Ende der 60er Jahre war es der $\gg$ heiße Herbst $\ll$, in dessen Verlauf eine zuvor geschwächte Arbeiterbewegung mächtig auf die politische Bühne trat und ihre Forderungen nicht nur nach monetärer Besserstellung, sondern nach anderen Wohnverhältnissen, neuen Lebensbedingungen auf die Straße trug und bei deren Realisierung mitwirken wollte.

»Vogliamo tutto《 (»Wir wollen alles «), vor allem "participazione«, demokratische Teilhabe auf allen gesellschaftlichen Entscheidungsebenen vom Universitätsinstiut, dem Stadtteilrat bis zu Mirafiori, jener FIAT-Fabrik, in der der »operaio di massa (der »Massenarbeiter $\ll$ ) nach Autonomie verlangte. »Arbeiterautonomie« wurde denn auch eine Forderung, die sich wie ein Lauffeuer in Westeuropa verbreitete, zumindest in Intellektuellenzirkeln und Kreisen linker Gewerkschafter, die nun anfingen, die grundlegenden italienischen Texte zu studieren - von Gramscis Analysc der Turiner Fabrikräte zu Beginn der 20er Jahre bis zu Rossana Rossandas oder Lucio Magris Reformismus-Kritik oder den Flugschriften von Potere operaio und Lotta continua. Der »Fall Italien« war in jener Phase leicht zu umschreiben: Die besondere Militanz der Arbeiterkämpfe, in denen die Gewerkschaften und die Parteien mitgerissen wurden, in deren Verlauf neue begeisternde Ideen geboren wurden, neue Institutionen und Erfahrungen entstanden, machte Italien zu einem »Fall« für die europäische Linke.

Druck erzeugt Gegendruck und die »politische Klasse« Italiens begann sich gegen die Zumutungen der Arbeiterkämpfe zu sammeln. Dabei spielten die alten Traditionen, die noch aus der Zeit vor dem Risorgimento stammen, eine Rolle, also Mafia und clandestine Organisationen wie die Freimaurerlogen (vgl. den Beitrag von Luciano Violante in diesem Heft), von denen die »loggia massonica des Licio Gelli (die »P2《) besonders berüchtigt geworden ist, zählte sie zu ihren Mitgliedern doch alles, was in Gesellschaft und Politik Italiens Rang und Namen hatte und hat von Geheimdienstlern, Armeegenerälen und hohen Regierungsmitgliedern bis zu dem Unternehmer Berlusconi, der freilich in jener Phase der 70er Jahre als politischer Akteur noch unbedeutend war. Selbst Putschversuche wurden in jener Phase angezettelt, erfolglos. In der linken Strategie reflektierte sich diese Gegenreaktion der »politischen Klasse«, denn sie konnte trotz aller Militanz nicht ignoriert werden.

Enrico Berlinguer, der damalige Vorsitzende der Kommunistischen Partei Italiens, entwickelte 1973 das der gesamten europäischen Linke eine Debatte aufnötigende Projekt des »Eurokommunismus « (Vgl. dazu Z.B. PROKLA $26,27,28,29,32)$, das die Verteidigung demokratischer Institutionen (deren Bedeutung für eine linke Reformpolitik durch den blutigen Putsch in Chile vor Augen geführt worden war) ebenso enthielt wie die prinzipielle Anerkennung kapitalistischer Verhältnisse, um sie einem demokratischen Reform- 
prozeß zu unterziehen. »Hegemoniegewinn« wurde zu einem Schlagwort, das - vielleicht in einseitiger Lektüre von Gramsci übernommen - in den politischen Diskursen von Berlinguer in Italien über Carillo in Spanien bis zu Glotz in der BRD Verwendung fand.

In der politischen Praxis, zumal angesichts der besonderen ökonomischen Schwierigkeiten Italiens, waren Kompromisse notwendig - bis zur Duldung (das »Nicht-Mißtrauen« - non-sfiducia) christdemokratisch geführter Minderheitsregierungen durch die größte linke Kraft, die Kommunistische Partei. Die Gewerkschaften wurden dadurch natürlich provoziert, denn nach den materiellen und politischen Erfolgen in den $»$ Klassenkämpfen in Italien « in der ersten Hälfte der 70er Jahre war nun »Austerità« als Regierungsprogramm angesagt, der »stangata« (Knüppelschlag), mit dem die direkten und indirekten Arbeitskosten zur Verbessenung der Konkurrenzfähigkeit gesenkt werden sollten. Italien gehörte zu einem der wenigen Industrieländer, die sich in den 70er Jahren (wie später in den 80er Jahren die verschuldeten Entwicklungsländer) einer IWF-Kur der »Strukturanpassung« unterziehen mußten. Erst zu Beginn der 90er Jahre wurde die bittere Medizin nicht mehr von den Medizinmännern aus Washington verabreicht, sondern von »Regierungen der Fachleute $\ll$, die sich weitgehend parteiungebunden und daher ohne Rücksichtnahme auf zivilgesellschaftliche Interessen als Experten der »Sachzwänge« aufspielen konnten. Ihnen war die Vertiefung des europäischen Integrationsprozesses hiflreich. Denn nach den Regeln des 1979 beschlossenen Euro- päischen Währungssystems war Italien zu einer deflationären Politik verpflichtet, für die in erster Linie die Gewerkschaften mit Lohnbegrenzungen zahlen mußten. Es hat lange gedauert, bis alle institutionellen Hindernisse einer Lohnflexibilisierung, vor allem die in der unmittelbaren Nachkriegszeit erkämpfte Lohngleitklausel (»scala mobile«), beseitigt worden sind (vgl dazu den Beitrag von Vittorio Rieser).

Der »Caso Italiano« hat also mit der viel größeren politischen Reaktionsgeschwindigkeit auf soziale und ökonomische Tendenzen zu tun als sie in anderen »normalen« (west)europäischen Ländern üblich ist. Dies zeigt sich auch bei dem Ereignis, das die Koordinaten der Politik in aller Welt veränderte: beim Fall der Berliner Mauer (darauf verweisen mehrere Autoren in diesem Heft) und dem Kollaps des »realen Sozialismus«, beim Verschwinden der von der »kommunistischen Tageszeitung« Il Manifesto so bezeichneten - »postrevolutionären Gesellschaften « von der historischen Bildfläche. Schon 1989, vor dem Fall der Mauer, hat Achille Occhetto, der damalige Vorsitzende der Kommunistischen Partei, eine Namensänderung und eine Reorganisation der KPI (zur »Partei der demokratischen Linkcn« - PDS) cingclcitct (vgl. dazu den Artikel von Marco Piantini). Dieser Kraftakt war nicht unumstritten. Denn die KPI war nicht nur die gößte westeuropäische KP, sondern auch die am wenigsten durch die Entwicklungen in Osteuropa kompromittierte kommunistische Partei, hatte sie doch die Unterdrückung in Prag 1968, in Kabul 1979 oder in Polen 1981 mit wünschenswerter Deutlichkeit kritisiert und sich seit den Tagen Togliattis 
um Autonomie gegenüber Moskau bemüht. Aus der Retrospektive betrachtet war der Schritt zur PDS richtig, allerdings in der Art der Durchführung glücklos und letztlich inkonsequent. Denn die PDS hat es nicht vermocht, mit der Reorganisation die Glaubwürdigkeit der Partei zu steigern und die eigene Erneuerung als Teil einer Strategie der Erneuerung der italienischen Gesellschaft einsichtig zu machen. So blieb der Akt der Umbenennung isoliert und politisch innerhalb und außerhalb der alten KPI und neuen PDS angefochten. Die Reorganisation der KPI zur PDS führte daher zu einer Abspaltung der »Rifondazione comunista $\ll$ (Kommunistische Emeuerung). Diese Partei ist im Gegensatz zur journalistischen Botschaft von FAZ bis TAZ keine sektiererische, fundamentalistische oder nostalgische Gruppe, sondern ein Kristallisationspunkt linker Opposition mit durchaus beachtlichen Wahlerfolgen. Die Wandlung der KPI zur PDS wurde aber nicht wirklich ernst genommen. Daher konnte es Berlusconi in den Tagen seiner Krise Ende 1994 wagen, den alten antikommunistischen Trumpf gegen die PDS zu ziehen, der eigentlich längst aus dem politischen Spiel sein sollte. Der Antikommunismus, ist anscheinend lebendiger als der Gegenstand der törichten Abneigung.

Der »Caso Italiano« erschöpft sich freilich nicht in der größeren politischen Volatilität, die niemals politische Langeweile aufkommen läßt. Die Besonderheit des »caso italiano« läßt sich am ehesten als Gegensätzlichkeit in der Einheit umschreiben. Wer die Filme über "Don Camillo und Peppone« gesehen hat, kann sich diese Gegensätzlichkeit sinnlich vorstellen: Katholizismus gegen Kommunismus und umgekehrt. Doch beide entstammen nicht nur der gleichen Kultur, sie sind auch in der jeweils anderen Identität präsent. Der italienische Laizismus wäre ohne die katholische Kultur und die Existenz des Vatikans nicht vorstellbar. Norden gegen Süden und umgekehrt. Der Mezzogiorno als Erbschaft seit der italienischen Einigung in den $60 \mathrm{er}$ Jahren des vorigen Jahrhunderts. Unterentwicklung im Süden und Modernisierung im Norden (was ubrigens nicht immer so war) - und zugleich ein »meccanismo unico«, ein einheitlicher Mechanismus, der beider Entwicklung in aller Gegensätzlichkeit steuert (siehe dazu den Beitrag von Isaia Sales). Dies mag ja ein Grund dafür sein, daß die Lega Nord seit den 80er Jahren und verstärkt seit Beginn der 90er Jahre den Versuch macht, sich des Südens zu entledigen, eine eigene territoriale Identität auszubilden (dazu der Beitrag von Gianni D'Amato und Siegfried Schieder in diesem Heft), also den »meccanismo unico« ein für allemal zu zerstören. Dabei könnte allerdings die Einheit, die Identität Italiens auf der Strecken bleiben. Ein rassistisch unterlegter Regionalismus im Norden würde wohl im Süden vergleichbare Antworten provozieren. Das müßte nicht wie auf dem Balkan Bürgerkrieg bedeuten. Aber das Zusarnmenleben südlich der Alpenbarriere auf der AppeninHalbinsel würde komplizierter werden.

Dann gibt es aber auch den Gegensatz zwischen der ineffizienten Staatsindustrie, die inzwischen weitgehend restrukturiert, liquidiert oder privatisiert worden ist, der modernen privaten 
Großindustrie der Agnelli, Olivetti oder Berlusconi und dem in modernen sozialgeographischen und industriesoziologischen Untersuchungen so sympathisch gezeichneten »terza Italia $\aleph_{\text {, jenen }}$ flexibel spezialisierten Klein- und Mittelunternehmen der sindustrial districts«, die auf dem Weltmarkt Furore machen. Tatsächlich ist diese Gegensätzlichkeit in der italienischen Wirtschafts- und Gesellschaftsstruktur eingeschrieben. In ihr werden synergetisch wirkende Ingredienzien zu einer Einheit gemischt, die für den über die Grenzen Italiens bewunderten ökonomischen Erfolg der 80er Jahre verantwortlich sind. Doch was für ein Erfolg war es, der Italien gegen Ende der 80er Jahre zur fünftstärksten Industrienation, sogar vor Großbritannien rangierend, aufblühen ließ3?

Wer in den 60er und 70er Jahren Italien kennengelernt hatte und erst wieder in den 80er Jahren das Land erneut besuchte, mußte sich auf einen anderen Stern versetzt fühlen. Einen wissenschaftlich abgeklärten, reflektierten Eindruck dieses unerhörten Wandels in Italien vermittelt der Aufsatz von Paul Ginsborg in diesem Heft. Weniger abgeklärt, dafür direkter wäre der Zeitsprung aus einer umbrischen Taverna in den frühen 70er Jahren, in das schicke und teurc Restaurant, in das sich die einstmalige Taverna in den 80er Jahren verwandelt hat. Noch deutlicher würde der Kulturbruch, wenn man den Besuch einer »festa dell'unità E Ende der 60er und Ende der 80er Jahre vergleicht. Der Vergleich würde einen Kulturbruch zutagefördern. In dem anscheinend so »ruhigen Jahrzehnt« der 80er Jahre hat zunächst unterirdisch und kaum bemerkt, dann eruptiv zu Beginn der 90er Jahre an die Oberfläche tretend, ein fundamentaler sozialer und kultureller Wandel stattgefunden: Der Wandel von einer sehr traditionellen, langsamen noch von agrarischen »Residuen« durchzogenen zur modernen, »schnelllebigen « und von der Industrie (auch auf dem Lande) geprägten Gesellschaft. In ihr mußte Kontinuität weniger als Ausdruck der sozialen Stabilität denn als Zeichen der Krise wahrgenommen werden. Alte Bindungen (daher spricht Franco Cazzola in seinem Aufsatz von »religio«) wurden von den Strömungen der modernen Konsumgesellschaft unterspült, mehr und mehr aufgelöst. Selbst die politische Moral der »resistenza«, die bislang für den »Verfassungsbogen $\ll$ unantastbar schien, wurde in $z$. T. perfider Weise in Frage gestellt, wenn die Unterscheidung zwischen Opfern (Widerstandskämpfer) und Tätern (Faschisten) aufgelöst wird. Dabei taten sich gerade Leute hervor, die sich im Zuge der Modernisierung von linken Traditionen zu befreien, die Nachkriegsgeschichte revisionistisch neu zu schreiben und faschistische Dichter und Denker als »Faszinosum« zu entdecken bemühten. Das kennen wir ja auch aus der bundesdeutschen Debatte der $80 \mathrm{cr}$ Jahre.

Es ist freilich vergleichsweise leicht, Werte, Orientierungen, moralische Prinzipien über Bord zu werfen, doch unendlich schwer, neue nicht nur intellektuell-diskursiv zu erzeugen, sondern lebenspraktisch und daher »ver-bindlich« (im Sinne Cazzolas Begriff von »religio« oder den »Ligaturen« Dahrendorfs) in einer Gesellschaft für Individuen zu verankern. Nur so könnten sich aus 
Leibniz'schen Monaden gesellschaftliche Individuen »emporarbeiten « - um den emphatischen begriff von Marx zu verwenden. Die italienische Modemisierung, mit so viel Verve im »terza Italia« eingeleitet, dürfte gerade an diesem Mangel aus dem Gleichgewicht geraten und daher von der Entwicklungsbahn abgekommen sein. Der Verlust der Moral liftete die Schranken gegen die öffentliche und private Korruption, die Schlamperei und die primitive Bereicherungsenergie. »Enrichissez vous « scheint das im Verlauf der 80er Jahren am meisten befolgte Gebot im »bel paese« gewesen zu sein, jedenfalls von einer groß- und kleinbürgerlichen Klasse, die keine Chance ausließ, um privat auch auf Kosten der Allgemeinheit zu akkumulieren. Italien hat eines der höchsten Budgetdefizite öffentlicher Haushalte in Europa (an die 10\% des BIP), eine öffentliche Verschuldung, deren Bestand etwa $125 \%$ des Bruttoinlandsprodukts ausmacht, den höchsten Anteil an Kapitaleinkommen am Volkseinkommen (über $40 \%$ ) in Westeuropa und Zinszahlungen der öffentlichen Haushalte, die bereits $10 \%$ des BIP beanspruchen. Da wird eine Klasse von Geldvermögensbesitzern durch die öffentlichen Haushalte alimentiert, die natürlich in allererster Linie Interesse an der Solvenz des Staates und daher an einer rigiden Sparpolitik hat natürlich nicht bei den Zinsen, sondern bei den »unproduktiven« Sozialausgaben. Die makro-ökonomischen Daten deuten mehr als eine Fiskalkrise an, sie verweisen auf eine Verschiebung der Klassenstruktur Italiens. Die Kämpen der Klassenkämpfe aus der heroischen Zeit des »heißen Herbstes« sind darin randständig geworden: die Industriearbeiter und ihre Organisationen, aber auch die »bodenständigen « Unternehmer-Kapitalisten. Wie in anderen Gesellschaften Westeuropas auch ist das Kapital transnational flexibler und mobiler als je zuvor. Die politische Steuerung wird mehr und mehr von den »Sachzwängen des Weltmarkts« durchkreuzt. Dies ist einer der Gründe, warum in Italien nach dem Berlusconi-Schock unter Dini ein Übergangskabinett der Experten gebildet wurde, das Sachzwänge exekutiert und dabei sogar auf einen relativ breiten Konsens der Parteien zählen kann, und warum als kommender Ministerpräsident, vielleicht als Führer einer neuen Mitte-Links-Koalititon Romano Prodi gehandelt wird, der äußere »Sachzwänge« und gesellschaftliche Interessen zu moderieren verspricht.

Das wird nicht leicht sein. Denn die zu bewältigende Krise der Staatsfinanzen ist die Kehrseite des Erfolgs der Kleinund Mittelbetriebe des vielgerühmten Terza Italia. Zweifellos hat das »dritte Italien « beachtliche technische und soziale Innovationen hervorgebracht, die die italienische Wettbewerbsfähigkeit in den vergangenen zwei Jahrzehnten gesteigert haben. Doch ein anderer Teil des Erfolgs beruht auch darauf, daß die Steuerehrlichkeit gering, die »evasione fiscale« groß und die Ausweichmanöver in Richtung prekärer Beschäftigungsverhältnisse verbreitet sind. Darüber schreibt Ginsborg in seinem Artikel in gebotener Klarheit. Dies ist eine für die Gesellschaft insgesamt pathologische »Entstaatlichung« und Privatisierung, eine Indienstnahme öffentlicher Ressourcen für private Zwecke. Transpa- 
rente Subventionen an die Unternehmen hätten ein Minimum demokratischer Kontrolle ermöglicht, die Art der Aneignung öffentlicher Mittel hat sie unterlaufen und dabei nicht nur die fiskalische Krise zugespitzt, sondern die öffentliche Moral unterminiert. Die politische Korruption, die von den Mailänder Staatsanwälten so mutig an den Pranger gestellt wurde, kam aus der Mitte der Gesellschaft, wenn auch weniger spektakulär.

Der Erfolg der kleinen und mittleren Unternehmen beruhte aber auch darauf, $\mathrm{da} B$ die gewerkschaftliche Organisation des »Massenarbeiters « in der »fabbrica diffusa $\ll$, in der in der Region zerstreuten Fabrik, unterlaufen werden konnte. Die tayloristisch-fordistische Organisation als »Massenarbeiter $\ll$ mit der $» G e-$ werkschaft der Räte«, die Bruno Trentin beschrieben hat, hat in den industrial districts des terza Italia ihren sozialen Ort verloren. Die Gewerkschaft müßte sich reorganisieren. Wie mühselig dies ist, zeigt die Reformdebatte nicht nur in Italien, sondern auch hierzulande. Die Schwierigkeiten werden noch durch die Veränderungen der Branchenstruktur (in Richtung Dienstleistungen) und der Altersstruktur (Zunahme der Rentner) erhöht. Die Arbeiterbewegung, die seit Ende der 60er Jahre so stark aufgetrumpft hat, ist in diesem »Mahlstrom « von Modernisierung, Tertiarisierung und Dezentralisierung, von Individualisierung und Konsumismus fast bis zum Hals versunken. Ein Mahlstrom ist dadurch gekennzeichnet, daß man umso tiefer in ihn hinuntergezogen wird, je mehr man sich darin Halt suchend bewegt. Wer selbst keinen festen Boden unter den Füßen lrat, wird anderen gesell- schaftlichen Akteuren kaum überzeugende Alternativen anbieten können. Wie in anderen europäischen Ländern auch haben Gewerkschaftsbewegung und politische Linke in Italien noch kein Rezept gefunden, wie aus dem Mahlstrom auf festen Grund zu gelangen sei, von dem aus die notwendigen gesellschaftlichen Veränderungen hätten vorwärtsgebracht werden können. Im Mahlstrom ist jeder allein. Die italienische Gesellschaft hat in diesen »ruhigen 80er Jahren «, in denen unter Craxis Schirm das Empire der Fernsehschirme des Silvio Berlusconi zusammengekauft werden konnte, die »piazza« verloren. Was dies heißt, kann jeder ermessen, der miterleben konnte, wie sich jede spiaggia in eine piazza verwandelte. Italien war selbst im Sommerloch eine durch und durch urbane und kommunikativ interessierte Gesellschaft. Individualisierte Individuen im Konsumismus brauchen die piazza jedoch nicht mehr als Ort der Begegnung, des Austausches, des Klatsches. Sie sind das gefundene Fressen für Medienhaifische wie Berlusconi. Die Gesellschaft ist schon lange vor dem überwältigenden Wahlsieg Berlusconis reif geworden für einen »brutalen«, vergewaltigenden Zugriff. Das virtuelle TV ersetzt die reelle piazza. Die monopolistische Manipulation untergräbt die populare Kommunikation und die demokratische Öffentlichkeit der piazza. Berlusconi der Held einer virtuellen Welt.

Freilich ein tragischer Held. Denn die Herrschaft kann nicht vollständig sein. Eine noch ausstehende gründliche Analyse der kurzen Amtszeit des Silvio Berlusconi wird - neben vielen anderen As- 
pekten - wahrscheinlich zu Tage fördern, daß es erstens auch mit der massivsten Medienmacht nicht möglich ist, eine Gesellschaft wie ein Unternehmen auto-, medio- und plutokratisch zu führen, daß zweitens das Trauma der Niederlage der Gewerkschaften, der Linken (im weitesten Sinne) nur temporär ist und der Widerstand gegen den Autokratismus einer Regierung und die virtuelle Welt von High Definition-TV heranreift und ausbricht, wenn die Zumutungen der realen Welt »über die Hutschnur « gehen - wie im Falle der zur Haushaltssanierung geplanten Kürzungen bei Pensionen und anderen Sozialleistungen im November 1994, daß drittens das politische Management anders als das Management eines Unternehmens der Legitimation vor kontroversen Interessen bedarf, die immer wieder neu erworben werden muß, daß viertens Mediokratie keine Aufbauhilfe für eine Regierungskoalition sein kann, die schon wegen der Mediokrität ihrer Protagonisten ins Chaos gerät. Freilich ist es überhaupt nicht ausgeschlossen, daß die von Berlusconi zusammengeführte Truppe lernt und beim nächsten Versuch nicht die Fehler des ersten Versuchs wiederholt. Denn auch in Italien ist die Rechte eine nicht $z u$ unterschätzende reale gesellschaftliche Kraft, die durch die transmittierten virtuellen Medienwelten nicht erzeugt, sondern nur gestützt wird.

Der Kurs der kommenden italienischen Regierungen ist vorgegeben, er heißt Europa. Europa, das sind die Kriterien von Maastricht und Maastricht verlangt von Italien eine radikale Sanierung der Staatsfinanzen. Wenn dies den technokratischen Regierungen nicht gelingt, wird Italien aus dem »Kerneuropa« herausfallen. Ganz neue Orientierungen müßten entwickelt werden, für die es keine Blaupause gibt. Auch die Linke ist strategisch und in der politischen Tagespraxis gefordert. Es gilt zu zeigen, wie die zweifellos notwendige Umstrukturierung von privater Ökonomie und öffentlichen Finanzen sozial verträglich und demokratisch mobilisierend vollzogen werden könnte. Die Linke... Damit kann im zusammenwachsenden Europa freilich nicht nur die italienische gemeint sein. Irgendwie ist der »caso italiano« ein »caso europeok. 